The Sustainable City XII 255

\title{
THE IMPORTANCE OF DESIGN IN HELPING HUMANITY BECOME A MULTI-PLANETARY SPECIES
}

\author{
CAMILO CERRO \\ College of Architecture, Art and Design, American University of Sharjah, UAE
}

\begin{abstract}
The United Nations projects that the world population will likely reach 10.9 billion by the end of the century on a planet ill prepared to provide shelter, health care and food and water for its inhabitants. To decrease the planets population, ensure the long-term continuation of our species and the survival of our evolutionary branch, humanity needs to become a multi-planetary species. By going off world to colonize the solar system, the first explorers and the later colonizers will need to inhabit environments designed specifically to preserve their psychological wellbeing. Developing design parameters based on psychological factors will be of paramount importance to help humans deal with long journeys through space, and to create relaxing habitats to help decrease the stress that planetary explorers will be subjected to. The design community needs to become a participant in this process because solely engineered spaces are not enough to develop a good mental and physical quality of life for space exploration. There has been a tendency, with few exceptions, to not include design as an important aspect of the development of the government funded space programs all around the world. It was not until private capital started participating on the field and saw the opportunity to involve the public, that designers were invited to help sell the ideas of space exploration. In the future, the participation of the designer will need to transcend the role of the beautifier and we will need to become involved in all aspects of design that relate to the psychological wellbeing of the explorers. The development of offplanetary architecture will help humanity transition and mentally adapt to foreign environments. We need to understand that off-world habitats will require design that conveys familiarity to aid with our survival and prosperity, but more importantly, to remind us where we came from.
\end{abstract}

Keywords: sustainability, multi-planetary species, mars colonization, space exploration, off-planet design, quality of life, mental health.

\section{INTRODUCTION}

"Since, in the long run, every planetary civilization will be endangered by impacts from space, every surviving civilization is obliged to become spacefaring--not because of exploratory or romantic zeal, but for the most practical reason imaginable: staying alive... If our long-term survival is at stake, we have a basic responsibility to our species to venture to other worlds." (Sagan [1]). And to do so, humanity will need habitats designed to help with the mental stress presented by the detachment from the planet that protects us. As humans leave home, we will need to be mentally prepared to endure the journey. This paper is a manifesto of ideas on the importance of design, in helping humanity become a multi-planetary species. In it I will be discussing interior architecture; designed around the systemic interdependence of four concepts: The psychological effect of color and light, collective memory in smart design, virtual reality immersion and mindfulness meditation. These four ideas will be the basis for a symbiotic hybridization in the creation of habitable environments designed for the purpose of calming the mind of the explorers that will spend a lot of time surrounded by none earth like landscapes or the vacuum of space. The habitable environments for space exploration and planetary colonization will need to stop resembling engineering labs like the international space station. Instead, they will need to look a lot like movie and TV science fiction sets, because we have become habituated to the stylistic representations created by the science fiction genre of what space design should be. Habitable 
space will need to be designed to calm the mind by developing familiarity and reminding us of home.

\section{THE PSYCOLOGICAL EFFECT OF COLOR AND LIGHT}

In the post-industrial era color and light have become important elements of design, helping; offices be more productive, fast food restaurants less appealing to linger in, emergency rooms less stressful, etc. But now, that man is starting to think about building habitats in outer space and other planets, the urgency of understanding the effect of artificial light becomes a necessity. Scientists have become convinced that light has a far greater impact on health and behavior than previously thought, making of it the most important environmental input, after food, in controlling bodily function. The use of color and light will be of special importance when dealing with life in other planets in helping us adjust our circadian rhythm (the Martian day is 24' 40", in Ceres it is 9'). The circadian rhythm is a roughly 24 hours cycle in the physiological processes of living beings, including plants, animals, fungi and cyanobacteria. It is important in determining the sleeping, feeding, brain wave activity, hormone production, cell regeneration and other biological patterns of all animals, including human beings. Because of this, the spaces we inhabit will need to be designed to help us cope with adapting our earth day to the new environments. This adaptation is paramount for our mental wellbeing and ultimately our capacity to travel to and colonize other planets. 'Architectural lighting must be redesigned to account for its biological and behavioral impact on humans, ultimately improving people's health and well-being in the built environment' (Holzman 2010 [2]). The habitats we design will need to have color changing light-emitting diodes (LEDs) serving as a natural stimulant which have the capacity of directly improving alertness, performance, and also helping reset an astronaut's circadian rhythm if it gets out of sync. The light in all sleeping habitats will need to be shifting from blue to white to red helping simulate the typical day to night cycle. The blue lighting is meant to stimulate the retinal photopigment melanopsin as well as the hormone melatonin, which helps a person feel alert and awake. The shift to red lighting will reverse the process and help encourage feelings of sleepiness. The rest of the habitats will be flooded with white light making the spaces easy to interact with. The versatility of the LED in color changing will also allow for other types of phototherapy to be implemented as needed. Green as an example, is said to lessen depression, anxiety, and nervousness. It relaxes both body and mind, and is both soothing and refreshing.

\section{COLLECTIVE MEMORY AND SMART DESIGN}

When we look at the international space station (ISS) or any of its predecessors we are confronted with utilitarian design constrained by budget, trade-offs, and practicality. We don't yet have the technology to do construction in space, so we have to assemble a large vehicle from launch able components. The components size and shape is dictated by the type of delivery vehicle. In the case of the ISS, all the components needed to fit in the Space Shuttle Orbiter or the Russian Proton rocket. The result is a series of very pragmatic exterior and interior design choices. But as we embark on long term exploration and the possibility of long term colonization, we need to consider the psychological effect of engineered spaces in the astronaut's mind. The interior design of this habitable spaces will need to answer to the collective memory of humanity. It will need to play with our nostalgia. And to do so, it will need to attach itself to universal interpretations of our understanding of what life in space should be like. This universal understanding comes from science fiction. Since Jules Verne, humanities collective memory has been creating an idea of what space inhabitation should be. This idea has evolved into the stage design of the movies and TV shows we watch. And 
it is these multiple representations, that make our generic collective understanding of interior environments something we find familiar. The interior design of our inhabitation modules and interior spaces will benefit from using this familiarity as a design tool that through association can generate spaces subconsciously acceptable to all. We need to make our habitable spaces look like our science fiction because it is this science fiction that has sparked in humanity the craving for exploration.

\section{VIRTUAL REALITY IMMERSION}

For explorers and colonizers open space will always be a luxury. In the colonial habitats and the spaceships, it will be impossible to physically reproduce the feeling of openness that we experience on earth. Psychologically, this feeling of constrain created by small spaces or being inside an environmental suit will eventually have a toll on the human mind.

A solution to this issue can come from the use of 360 virtual reality immersion as a tool for relaxation. For the purpose of this argument we will define VR as "an advanced form of human-computer interface that allows the user to interact with and become immersed in a computer-generated environment in a naturalistic fashion" (Schultheis and Rizzo [3]). Presently this technology is applied in the treatment of phobias, anxiety, depression, PTSD, obesity and eating disorders, and also purely for relaxation purposes.

Off earth, this tool would present an easy way of dealing with our nostalgia for the world left behind. The technology is small, which allows the user to get immersed anywhere. But most importantly, the immersion scenarios are unlimited: These include landscapes, video games, tutorials or personal interactions with family and friends. Because the technology is therapeutic, didactic and entertaining, it will end up as a participant in space exploration helping define quality of life in all off world endeavors.

\section{MINDFULNESS MEDITATION}

"Although the practice of meditation is associated with a sense of peacefulness and physical relaxation, practitioners have long claimed that meditation also provides cognitive and psychological benefits that persist throughout the day" (Lazar [4]).

Studies have found structural differences between the brains of experienced meditation practitioners and individuals with no history of meditation, observing thickening of the cerebral cortex in areas associated with attention and emotional integration. The study by
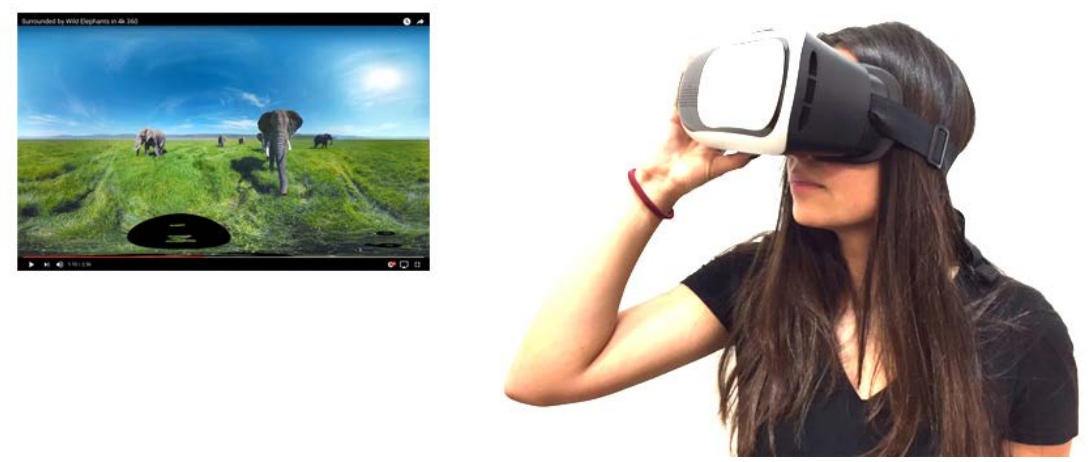

Figure 1: VR immersion headset will allow the user to escape the isolation of their physical situation. 
Lazar and associates shows that the brain's plasticity under meditation can play an active role in changing the brain. By doing so, increasing our well-being and quality of life, results which would be psychologically beneficial to off world explorers and colonizers. A controlled mind would minimize mental health problems and would allow for social harmony and adaptability to the new environmental constrains. Tibetan Buddhist monks go on three-year retreats to caves on the side of mountains in the Himalayas, not leaving the cave for the extent of the retreat. This level of mental control has the potential to define the success of space exploration. In my mind, astronauts will need to be good meditators before going off world, and their meditation practice should be carried through the extent of their deployment. A focused and balanced state of mind would allow for a level of behavioral equanimity necessary in the social interaction of small communities that have to function together for extended periods of time.

\section{METHODOLOGY}

In the study of interior architecture for the exploration and colonization of our solar system, a series of interior space related proposals were designed as an attempt to provide a basis to address all the issues covered previously. These proposals will be explained in point 8 . But it is important to note that the research of this subject has been an ongoing process which as a side effect has produced an independent study course at an undergraduate architecture program. The course was developed to test some of this research in an academic context but the ultimate goal of this work is implementation. To explain the research process, the following methodology was produced:

- An ongoing analysis of different literature sources was established to define trends and features related to the influence of psychology in the development of interior design for off planet habitation, focusing on design approaches taken by NASA, ESA, Virgin Space and Space X.

- Case studies of previous design proposals produced by other designers where studied.

- An independent study was set up with a fifth-year undergraduate student at the American University of Sharjah. The research project carried on for two semesters and was presented as his final thesis. Point 7 will explain the independent study.

- By defining possible micro sites, questions of; the psychological effect of color and light, collective memory smart design, virtual reality immersion and meditation, were studied and assessed.

- A series of design were proposed so they could be reviewed by peer designers.

- 3D Models were produced to help convey some of these ideas representationally.

\section{STUDENT WORK: INDEPENDENT STUDY}

To further explore some of the ideas presented on this paper a one-year independent study was approved by the American University of Sharjah with the aim of implementing this research in a design studio. Some samples of the work generated by the student are presented below. The studio was set up as an alternative to the typical fifth year studio. In it one student would work with a mentor to implement faculty research. The studio was set up as an end of degree thesis in which the fall semester would be used by the student to produce research. The research would be presented at the end of term in front of a jury that would determine if there was enough merit for the project to continue during spring semester. The final thesis project was presented in the form of a comic book that explored through a narrative all the material researched over the year. While the level of development does not take into 
consideration a lot of the constrains presented by the task of creating habitat for mars colonization, the studio was successful in opening a dialogue on the subject at an academic level. It also was successful at helping clarify a path for the research that came after the independent study. That research is being presented in this paper.
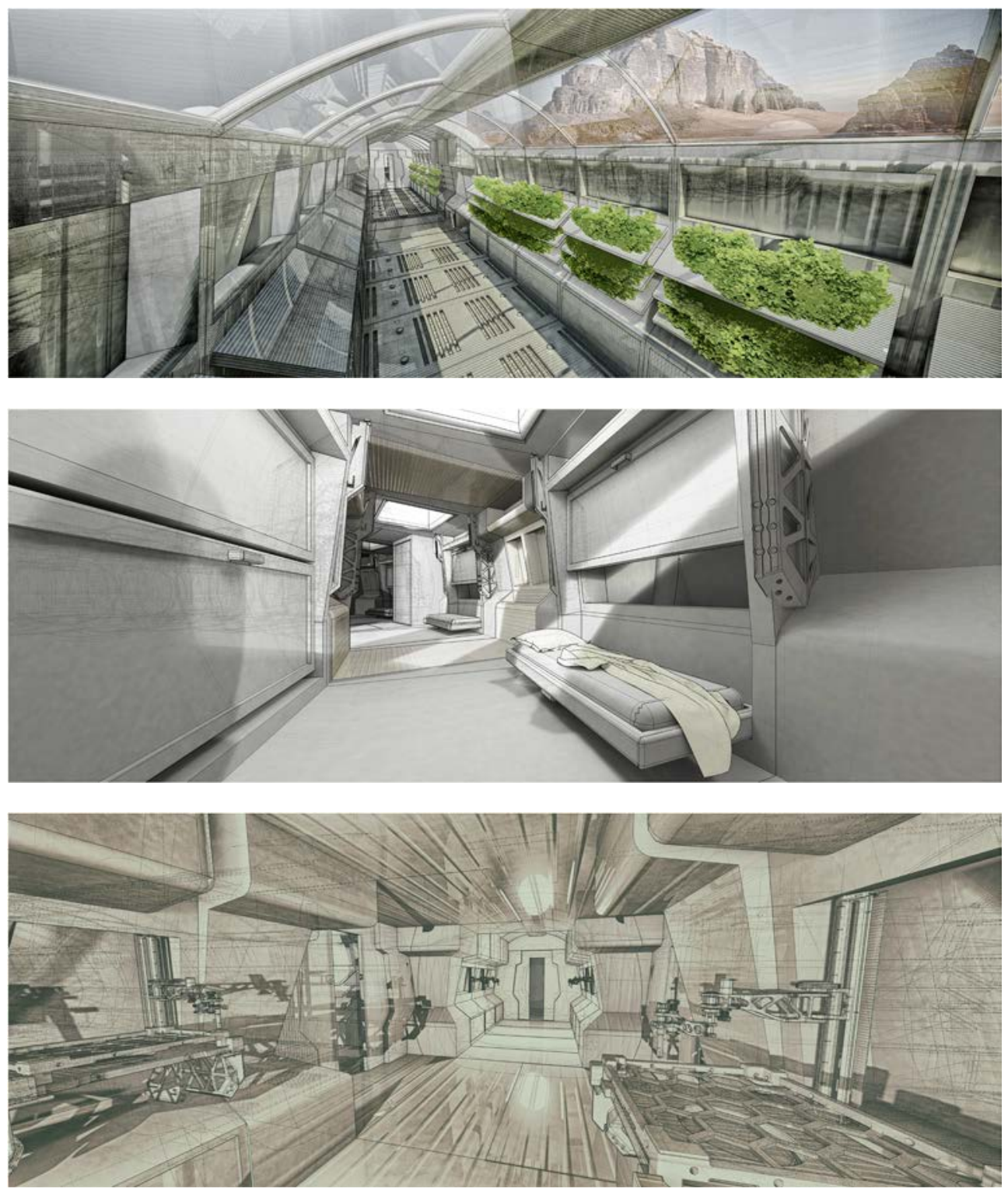

Figure 2: Work produced by Al Chawa [5]. 
8 CASE STUDY: MARS COLONY HABITAT SPATIAL DESIGN

The main premise of the case study was to hybridize the conceptual elements previously presented: The psychological effect of color and light, collective memory in smart design, virtual reality immersion and mindfulness meditation, into interior architecture proposals. All the proposed spaces will deal directly with design elements that will have a psychological effect on the user. Plants for the production of oxygen and food will also add a visual component to the habitats linking the astronaut to earth while also offering activities like gardening as a distraction or hobby. Together with the plants, a water element is always part of the habitats, the water will be in motion for filtration purposes, allowing the design of the spaces to use the sound of water in specific locations to sooth and relax the users mind.

The first space is the connector hallway (Fig. 3) which is composed of a spinning hydroponic bay inside a circulation hallway. The modular ring is able to be expanded as needed altering the length of the hallway. Because of the lack of space, hallways need to serve more functions than just being circulation elements. That is why the interior of the walls are used for insulation, electric and life support systems. As the hydroponic bay spins, holes on the bottom of the hydroponic cylinders allow for the nutrient solution to be in contact with the plants. The hydroponic bay's speed cycle and type of nutrient solution are adaptable. And at any point, the nutrient solution can be filtered for water consumption. The proposal allows for all circulation hallways to also function as hydroponic farms, producing food and cleaning the air of the colony, while creating a visual relationship with the plants. The later one is important because maintaining a connection to nature, either through the presence of plants or artwork depicting the natural environment, has been shown to decrease stress levels and stimulate healing. The presence of plants in interior spaces has also shown to: Improve reaction times, attentiveness, attendance (at work and school), productivity (at work), well-being, perceptions of the space around you, lower blood pressure, lower levels of anxiety while recovering from surgery and better job satisfaction.

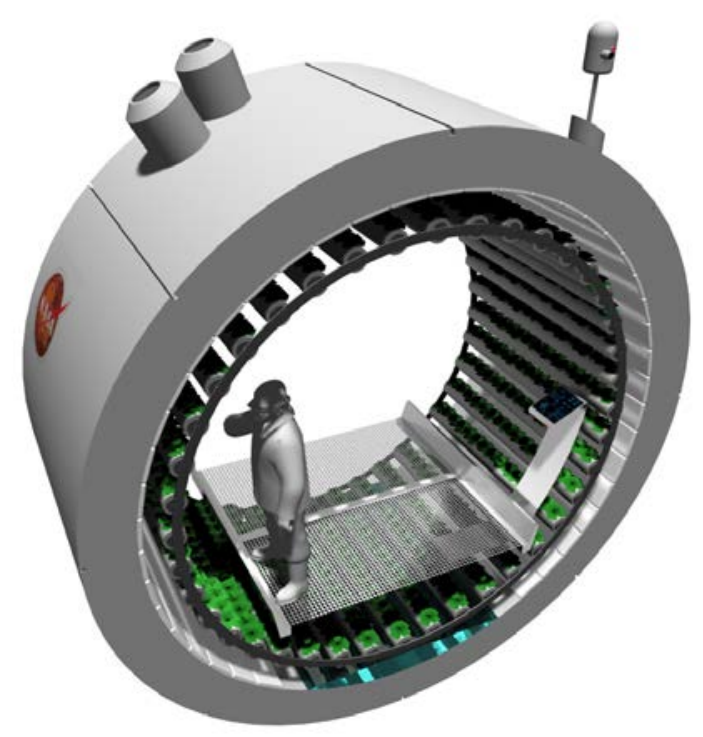

Figure 3: Connector hallway with hydroponic bay and LED growth lights. 


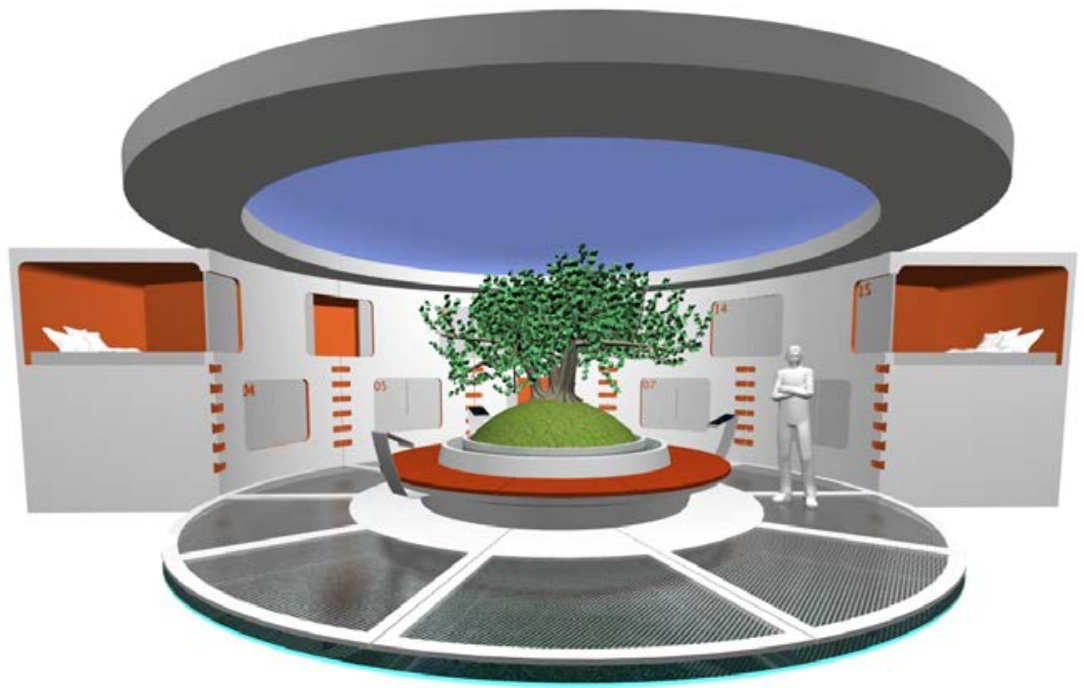

Figure 4: Sleeping and relaxation module with plants, water storage under main floor, circadian rhythm dome and private bunk beds for sleeping and meditation.

The second space is the sleeping module (Fig. 4), designed as a panopticon system of bunks that open to a shared central space. The bunks are designed to close off the central space to provide privacy. Each bunk has space for a bed that fits two, plus shelving and storage for personal items. The space functions similarly to a micro hotel. Opposite to the bunks the astronauts would have a shared bathroom and locker spaces. Each bunk cubby will have an illumination system that will link to the user's circadian rhythm, changing colors to simulate sun rise and sun set. The central space will also be linked to the stations general circadian rhythm to serve as an internal clock shared by the whole facility. The central shared space is composed of a seating area designed on top of a see-through floor system that covers one of the water holding units. The users will be able to see and hear the water. In the middle of the room a tree serves as a center piece to be seen at the beginning and end of each astronaut's day. The tree, the water and the light are specifically designed to produce psychological reactions that associate these lives bringing elements with the collective memory of the crew to subconsciously make them feel at home.

The third space is the food court (Fig. 5) which is designed around a central element that holds three stacking tables. The tables can move around the central element and because they are designed at different heights, they are capable of stacking together to allow for the space to have other uses. There is a hydroponic farm surrounding the main space which links to a water reservoir below the floor. The dome is designed to work around the stations general circadian rhythm.

You might have noticed that the interior surfaces of most spaces are white, this has been done to allow the light to control the coloration of the spaces instead of the surfaces. In a white surface, the light will reflect the given color manifested by the LED, instead of blending with an existing color. The purity of the color produced by the light will allow for the photobiology (color therapy) to function better by controlling the exact wavelength of the color needed. 


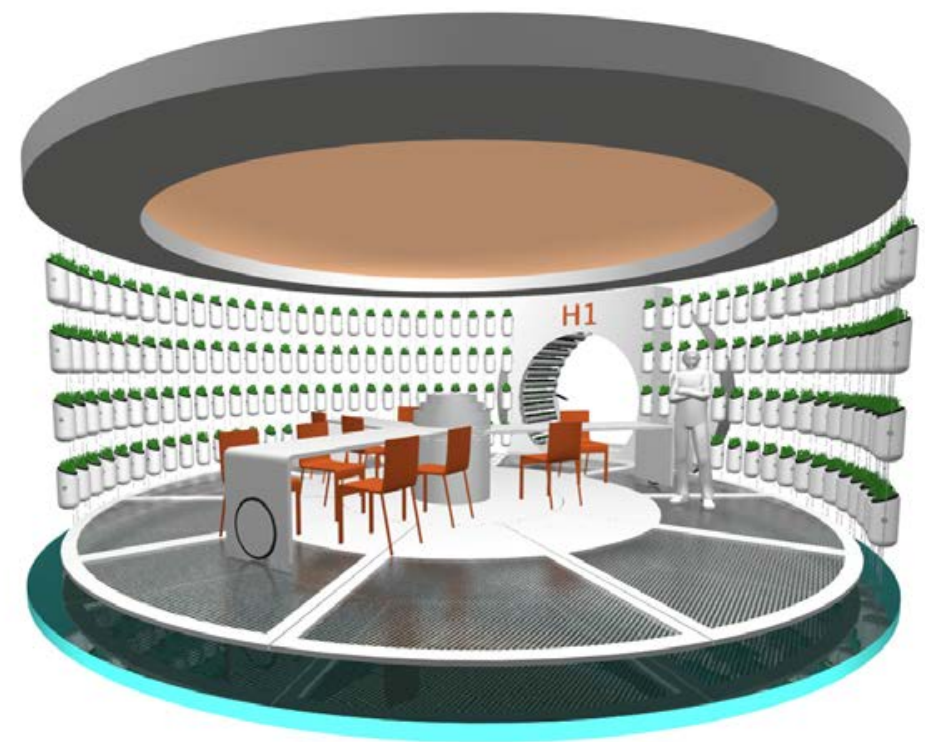

Figure 5: Food court with hydroponic food production, water storage under main floor, circadian rhythm dome and overlapping movable tables for adaptable space.

\section{CONCLUSION}

The aim of this project was to start a discussion about the role of psychological based design in the exploration and colonization of the solar system. By developing spatial proposals that deal with the psychological effect of color and light in habitats designed to engage humanities collective memory, we find an architectural framework that has the potential to create healthy environments to help resolve questions of quality of life while living away from earth. And by doing so, creates spaces where non-architectural psychological elements like virtual reality immersion and mindfulness meditation, can manifest. The spatial proposals offer:

- Inhabitation in spaces designed with an understanding of the psychological effects of color and light, which will produce less oppressive interior architecture and will help keep the astronauts in synchrony with their circadian rhythm.

- An architectural typology of designed spaces to serve the collective memory of humanity through the creation of familiar spaces that evoke a nostalgic memory of the home planet.

- The use of virtual reality immersion to help the crew relax and or to deal with mental health issues like depression or phobias.

- Sleeping spaces designed to provide some level of privacy and for the practice of mindfulness meditation.

When the four subjects explained in this paper develop a systemic interdependency, we end up with a series of proposals that manifest the importance that interior architecture has in helping us become a multi-planetary species. 


\section{ACKNOWLEDGEMENT}

This research was used to produce and independent study course at the American University of Sharjah. The course was divided into two semesters: The design studio course that took place in the Fall of 2015, had as a goal to produce research on possible landing sites, emerging technologies, existing designs, ergonomic design, energy, food, and transport. In Spring of 2015, the design studio course dealt with implementation. The studio had a single thesis student; Mohamad Al Chawa.

\section{REFERENCES}

[1] Sagan, C., Pale Blue Dot: A Vision of The Human Future in Space, Random House, Inc: New York, p. 371, 1994.

[2] Holzman, D.C., What's in a color? The unique human health effects of blue light. Environmental Health Perspectives, 118(1), pp. A22-A27, Online. http://www.jstor.org/stable/30249892. 2010.

[3] Schultheis, M.T. \& Rizzo, A.A., The application of virtual reality technology in rehabilitation. Rehabilitation Psychology, 46, pp. 296-311, 2001.

[4] Britta, K.H. et al., Mindfulness practice leads to increases in regional brain gray matter density. Psychiatry Res., 191(1), pp. 36-43, Online. doi: 10.1016/j.pscychresns. 2010.08.006, 2011.

[5] Mohamad, A.C., Atlas, Fifth year independent study at the American University of Sharjah, Mentored by Camilo Cerro, 2015. 